\title{
Spectral Analysis of Four Term Differential Operator
}

\author{
NyAMWALA FREDRICK OLUOCH \\ Fachbereich Mathematik/Informatik, Universität Osnabrück, 49069 Osnabrück, \\ Germany \\ e-mail : fonyamwa@mathematik.uni-osnabrueck.de
}

Abstract. By strengthening dichotomy condition and weakening decay conditions, we show that a four term $2 n$-th order differential operator with unbounded coefficients is nonlimit-point. Using stringent conditions we show that the deficiency index of this operator is determined by the behaviour of the coefficients themselves. Similarly, we prove the absence of singular continuous spectrum and that the absolutely continuous spectrum has multiplicity two.

\section{Introduction}

This paper is concerned with the study of deficiency indices and spectral results of a four term $2 n$-th order differential operator generated by a differential expression $\tau$ of the form

$$
\begin{gathered}
\tau y=w^{-1}\left\{(-1)^{n}\left(y^{(n)}\right)^{(n)}-\frac{1}{2} i\left(\left(p y^{(k+1)}\right)^{k}+\left(p y^{(k)}\right)^{(k+1)}\right)\right. \\
\left.+(-1)^{m}\left(q y^{(m)}\right)^{(m)}+p_{0} y\right\}
\end{gathered}
$$

defined on $\mathcal{L}_{w}^{2}([0, \infty))$. The coefficients as functions of $x$ are assumed to be realvalued, locally integrable, twice differentiable and are allowed to be unbounded. In the special case where $w \approx p_{0}$, then $q$ and $p_{0}$ will be assumed to be asymptotically constant, that is, $q \rightarrow c_{q}$ and $p_{0} \rightarrow d_{p_{o}}$ as $x \rightarrow \infty$ [2], where $c_{q}$ and $d_{p_{0}}$ are constants. The symmetric nature of this differential expression has been studied by Everitt and others in several papers, for example [11]. Just like in $[3,7,15,16]$, asymptotic integration theory will also be used here to study the deficiency indices and spectral results. Asymptotic integration theory of linear Hamiltonian systems is based on Levinson's Theorem [10]. There are many authors who have used asymptotic integration theory to investigate deficiency indices and spectral results of higher order differential operators $[8,10,9]$. This theory has also been extended to investigate absolutely continuous spectrum of differential operators by extending Levinson's

Received August 4, 2009; revised October 14, 2009; accepted November 20, 2009. 2000 Mathematics Subject Classification: Primary 34L05; Secondary 47E05.

Key words and phrases: differential operators, deficiency indices, absolutely continuous spectrum, eigenvalues. 
Theorem in various directions to include spectral parameter and also by weakening decay conditions $[2,7,15]$. The absolutely continuous spectrum is only stable under relative trace class perturbations. Previously, it has been studied mainly via scattering techniques. The singular continuous spectrum is much more elusive, because it is even unstable under small finite rank perturbations. This makes asymptotic integration unsuitable for the study of singular continuous spectrum and thus by results of [15], it will be absent in our case.

Eastham $[8,9,10]$ used asymptotic theory to derive deficiency indices of general even order differential operators although he assumed the coefficients to be once differentiable. Remling [15], extended this theory to study absolutely continuous spectrum of higher order operators although he could not deal effectively with unbounded coefficients since differential operators with unbounded coefficients may exhibit a non-limit-point-case. This problem was later resolved by introducing separated boundary conditions. Our main results states that a four term $2 n$-th order differential operator (1.1) with unbounded coefficients has non-limit-point behaviour, the deficiency index is the sum of deficiency indices within each eigenvalue group where the eigenvalue groups are determined by the growth of the coefficients themselves. The singular continuous spectrum is absent and the absolutely continuous spectrum has multiplicity two. These results are achieved through application of appropriate smoothness and decay conditions. Once the asymptotics of the eigenfunctions have been determined, the estimates for the $M$-matrix can be derived for the operators on the interval $[a, \infty)$ with $a$ sufficiently large. Results of Remling $[15]$ then allow one to extend these results to the interval $[0, \infty)$.

In order to associate $\tau$ to a minimal closed operator $L$ and maximal operator $L^{*}$, we make the following assumptions. The coefficients are real valued, locally integrable and $w>0$. The scalar product on the Hilbert space $\mathcal{L}_{w}^{2}([0, \infty))$ will then be defined with respect to weighted function $w$. Generalising the approach of Weidmann [18] and Eastham [10], we assume the coefficients to admit a decomposition of the form

$$
f=f_{1}+f_{2}+f_{3}+f_{4}, \quad f_{2}=o\left(f_{1}\right), \quad f=p, q, p_{0}, w .
$$

Here $f_{1}$ and $f_{2}$ are assumed to be twice, respectively once, differentiable. The sum $f_{1}+f_{2}=f_{s}$ will be called the smooth part of $f, f_{3}$ will be assumed to be integrable while $f_{4}$ is assumed to be conditionally integrable. Then define also $\widehat{f_{4}}=-\int_{0}^{\infty} f_{4}(t) d t$.

Since $p_{0}, q, p$ are real valued by assumption, $\tau$ on $\mathcal{L}_{w}^{2}([0, \infty))$ is formally symmetric and thus has self-adjoint extensions $H$ if the deficiency indices def $L$, of its minimal operator are equal. Thus $\sigma(H), \sigma_{a c}(H), \sigma_{e s s}(H), \sigma_{p}(H)$ and $\sigma_{s c}(H)$ will denote spectrum, absolutely continuous spectrum, essential, point and singular continuous spectrum of $H$ respectively.

$$
\operatorname{defT}=\left(\operatorname{dim} \mathcal{N}_{T^{*-i}}, \operatorname{dim} \mathcal{N}_{T^{*}+i}\right)=\left(N_{-}, N_{+}\right), \quad \mathcal{N}=\text { nullspace }
$$

will define deficiency indices. Note that different authors for example Eastham [10] and Weidmann [18] define deficiency indices differently. Thus in this paper, we will 
stick to the definition given above. The symbols $\gg$ and $\ll$ will be used to denote much larger than and much smaller than respectively, in the absolute value sense. Similarly, the relation $f \approx g$ will imply that $K^{-1}|f| \leq|g| \leq K|f|$ with $K$ a constant.

The paper is divided into four sections namely; 1. Introduction, 2. System Formulation, 3. Dichotomy Condition, 4. Spectral Results.

\section{System Formulation}

In order to write a general $2 n$-th order differential expression of the form

$$
\begin{aligned}
L y & =w^{-1}\left\{\Sigma_{k=0}^{n}(-1)^{k}\left(p_{k} y^{(k)}\right)^{(k)}\right. \\
& \left.-i \Sigma_{j=1}^{n}(-1)^{j}\left(\left(q_{j} y^{(j)}\right)^{(j-1)}+\left(q_{j} y^{(j-1)}\right)^{(j)}\right)\right\}
\end{aligned}
$$

into its linear Hamiltonian system or first order system, one needs to introduce quasiderivatives. For operators of the type $\tau$ (2.1), these have been defined by Walker [17]. With $n \geq 2$ in (2.1), and some obvious reindexing, $k \leftrightarrow n-k$, one has

$$
\begin{gathered}
y^{[k]}=y^{(k)} \text { for } 0 \leq k \leq n-1, \quad y^{[n]}=p_{n} y^{(n)}-i q_{n} y^{(n-1)}, \\
y^{[n+1]}=-\left(y^{(n)}\right)^{\prime}+i\left(q_{n} / p_{n}\right) y^{[n]}+\left(p_{n-1}-\left(q_{n}^{2} / p_{n}\right)\right) y^{(n-1)}-i q_{n-1} y^{(n-2)}, \\
y^{[n+k]}=-y^{[n+k-1]^{\prime}}+p_{n-k} y^{[n-k]}+i\left(q_{n-k+1} y^{(n-k+1)}-q_{n-k} y^{(n-k-1)}\right)
\end{gathered}
$$

for $2 \leq k \leq n-1$. Then for $n \geq 2$,

$$
\tau y=w^{-}\left[-\left(y^{[2 n-1]}\right)^{\prime}+i q_{1} y^{\prime}+p_{0} y\right]
$$

so that the domain of maximal differential operator $L^{*}$ generated by $(2.1), D\left(L^{*}\right)$, consists of all functions $y$ for which $y^{[k]}$ with $0 \leq k \leq 2 n-1$ are absolutely continuous and $L^{*} y \in \mathcal{L}^{2}([0, \infty), w)=\mathcal{L}_{w}^{2}$. Precisely, this domain is given by

$D\left(L^{*}\right)=\left\{y \in \mathcal{L}^{2}((0, \infty): w): \quad y^{[0]}, y^{[1]}, \cdots, y^{[2 n-1]}\right.$ are absolutely continuous in $\left.((0, \infty): w), \tau y \in \mathcal{L}^{2}((0, \infty): w)\right\}$

and $\tau y=L^{*} y$ for $y \in D\left(L^{*}\right)$. Thus, the maximal operator $L^{*}$ is defined in the largest possible domain in $\mathcal{L}^{2}((0, \infty): w) . \quad L^{*}$ is therefore densely defined and closed. An operator defined by restricting the domain of the maximal operator only to those functions $y$ with compact support is known as pre-minimal operator. This will be denoted by $L_{1}$ and its domain is defined by

$$
D\left(L_{1}\right)=\left\{y \in D\left(L^{*}\right): \quad y \text { has compact support in }(0, \infty)\right\}
$$

and $L_{1} y=\tau y=L^{*} y$ for $y \in D\left(L_{1}\right)$. The closure of pre-minimal operator, $\bar{L}_{1}$, is the minimal differential operator generated by (2.1) and will be denoted by $L$.

Thus the linear Hamiltonian system of (2.1) is given by

$$
\mathcal{J} y^{\prime}(x)=[z \mathcal{A}(x)+\mathcal{B}(x)] y(x),
$$


where $\mathcal{A}(x), \mathcal{B}(x)$ and $\mathcal{J}$ are matrices of size $2 n \times 2 n$ and $y$ is a function with values in $\mathbb{C}^{2 n}$ and satisfies regularity conditions, that is, if $y$ satisfies $\mathcal{J} y^{\prime}-\mathcal{B} y=z_{0} \mathcal{A} y$ for some $y$ and some $z_{0}$ with $\|y\|_{\mathcal{A}}=0$ then $y=0$, and $\mathcal{J} y^{\prime}-\mathcal{B} y=\mathcal{A} f$ with $\|y\|_{\mathcal{A}}=0$ then $\|f\|_{\mathcal{A}}=0$. Furthermore,

$$
\mathcal{J}=\left[\begin{array}{cc}
0_{n} & -I_{n} \\
I_{n} & 0_{n}
\end{array}\right], \quad \mathcal{A}=\left[\begin{array}{cc}
A_{1} & 0 \\
0 & 0
\end{array}\right],
$$

where $A_{1} \in \mathbb{C}^{s \times s}$ with an assumption that $\mathcal{A}(x), \mathcal{B}(x)$ are locally integrable in the underlying interval $[a, \infty)$ and that $\mathcal{B}(x)=\mathcal{B}^{*}(x), A_{1}(x)>0$ (in the positive definite sense), almost everywhere. It is this form of $\mathcal{J}$ which made the reordering of the last $n$ components of $\left(y^{[k]}\right)_{k=0}^{2 n-1}$ necessary. The nonzero matrix elements of $\mathcal{A}(x)$ are $\mathcal{A}_{11}=w$ while $\mathcal{B}=\left(\begin{array}{cc}-C & A^{*} \\ A & B\end{array}\right)$ with the nonzero matrix elements of $A, B$ and $C$ given by

$$
\begin{gathered}
A_{j, j+1}=1, \quad A_{n, n}=i \frac{q_{n}}{p_{n}}, \quad B_{n, n}=p_{n}^{-1}, \\
C_{j, j}=p_{j-1}, \quad C_{j, j+1}=i q_{j}=-C_{j+1, j} .
\end{gathered}
$$

Here, $p_{0}$ and $p_{n-1}$ should be read as $p_{0}-z w$ and $p_{n-1}-\frac{q_{n}^{2}}{p_{n}}$ respectively, where $z$ is the spectral parameter. Thus, the differential operator generated by $\tau$ is defined on the Hilbert space $\mathcal{H}_{A}=P_{s} \mathcal{L}^{2}([a, \infty))$ consisting of the equivalence classes of measurable $\mathbb{C}^{s}$-valued functions $f$ with

$$
\int_{a}^{\infty} f^{*}(x) \mathcal{A}(x) f(x) d(x)<\infty .
$$

In the definition of the Hilbert space $\mathcal{H}_{A}, P_{s}$ is used to denote the projection on the first $s$ components of a vector in $\mathbb{C}^{2 n}$. The scalar product is then given by

$$
\langle f, g\rangle_{A}=\int_{a}^{\infty} f^{*}(x) A(x) g(x) d(x) .
$$

On this space define the operator $\widetilde{\tau}$ formally by

$$
\widetilde{\tau} y=\operatorname{diag}\left(w^{-1}, 0, \cdots, 0\right)\left[\mathcal{J} y^{\prime}-\mathcal{B} y\right] .
$$

Then $\widetilde{\tau}$ is formally symmetric and one can define again the corresponding minimal and maximal operators $\widetilde{L}$ and $\widetilde{L}^{*}[13,14]$. However, it is advantageous to write (2.3) in its first order system. Assume that $u=\left(y^{[0]}, \cdots, y^{[n-1]}, y^{[2 n-1]}, \cdots, y^{[n]}\right)^{t}$, then one has

$$
u^{\prime}=\left(\begin{array}{cc}
A & B \\
C & -A^{*}
\end{array}\right) u=\mathcal{C} u .
$$

These two systems, (2.3) and (2.4), are unitarily equivalent and thus their spectral results are equal $[13,14]$. If $L$ is in limit point at infinity, that is, $\operatorname{def} L=(n, n)$, then 
the self adjoint extensions $H_{\alpha}$ are defined by boundary conditions at 0 parametrised by $n \times n$ matrices $\alpha_{1}$ and $\alpha_{2}$ with

$$
\alpha_{1} \alpha_{1}^{*}+\alpha_{2} \alpha_{2}^{*}=I_{n}, \quad \alpha_{1} \alpha_{2}^{*}=\alpha_{2} \alpha_{1}^{*} .
$$

Then $H_{\alpha} u=L^{*} u$ for $u \in D\left(H_{\alpha}\right)$, where

$$
D\left(H_{\alpha}\right)=\left\{u \in D\left(L^{*}\right) \mid\left(\alpha_{1}, \alpha_{2}\right) u(0)=0\right\} .
$$

We thus define a differential operator a $k$-term operator if besides the leading term and $p_{0}$, only $k-2$ other coefficients differ from zero. They will be referred to here as almost $k$-term operator if all but the $k$-coefficients of (2.1) vanish at infinity. Throughout this study, the deficiency indices and spectral analysis of the four term differential operator generated by (1.1) will be done in the formal framework of linear Hamiltonian systems or its equivalent, the first order system. On one hand, one can use the theory of general $M$-fuction, as developed by Hinton and Schneider $[13,14,15]$, to obtain spectral results. In [14], Hinton and Shaw and in [13], Hinton and Schneider have developed the spectral theory of differential operators which can be put into Hamiltonian form.

One of the most important results in asymptotic integration theory which helps in estimating the solutions of the first order system (2.4) is the Levinson's Theorem. It states that the solutions of a system

$$
u^{\prime}(x)=\{\Lambda(x)+R(x)\} u(x), \quad \Lambda(x)=\operatorname{diag}\left(\lambda_{i}(x)\right)
$$

look like the solutions of the unperturbed system $u^{\prime}=\Lambda u$, if $R(x)$ is sufficiently small and $\Lambda(x)=\operatorname{diag}\left(\lambda_{i}(x)\right)$ satisfy the dichotomy condition. In Levinson's original result, small means absolutely integrable. The dichotomy condition amounts to: $\operatorname{Re} \lambda_{i}(x)$ and $\operatorname{Re}\left(\lambda_{k}(x)-\lambda_{j}(x)\right), k \neq j$, have approximately constant sign modulo $\mathcal{L}^{1}$ for large $x$. Meanwhile, Levinson's Theorem has been extended in several directions by modifying the dichotomy condition as well as allowed perturbations. These permissible perturbations will be called Levinson's terms in order to simplify the use of Levinson's Theorem.

For more details, see the book of Eastham [10] or [6]. In spectral theory, the matrix elements and $\lambda_{i}(x)$ will generally depend also on the spectral parameter $z$. Thus one writes $\lambda_{i}=\lambda_{i}(x, z)$ for this. In this case, it will be important to prove Levinson's Theorem uniformly in $z$ in order to control the $z$-dependence of the solutions. The following version given in $[5,6]$ will suffice.

Theorem 2.1. Let $\Lambda(x, z)=\operatorname{diag}\left(\lambda_{1}(x, z), \cdots, \lambda_{2 n}(x, z)\right)$ and $R(x, z)$ be $2 n \times 2 n$ matrices which for a fixed $x$, are analytic functions of $z \in \Omega$ (where $\Omega$ is an open subset of $\mathbb{C})$ and

(i) For every compact subspace $K \subset \Omega$ there exists a function $f=f_{k} \in$ $\mathcal{L}_{\text {loc }}^{1}([a, \infty))$ with $\|\Lambda(x, z)\| \leq f(x), \forall z \in K, x \geq a$,

(ii) $\operatorname{Re}\left\{\lambda_{i}(x, z)-\lambda_{j}(x, z)\right\}$ of $[a, \infty) \times \Omega$ for all pair of numbers $i$ and $j$ in $(1, \cdots, 2 n), i \neq j$, have constant sign modulo $\mathcal{L}^{1}$ (Dichotomy condition), 
(iii) $\|R(x, z)\| \leq g(x)$ with $g \in \mathcal{L}^{1}([a, \infty))$,

then the system

$$
Y^{\prime}(x, z)=[\Lambda(x, z)+R(x, z)] Y(x, z)
$$

has solutions of the form

$$
Y_{k}(x, z)=\left(e_{k}+r_{k}(x, z)\right) \exp \left(\int_{a}^{x} \lambda_{k}(t, z) d t\right)
$$

with $r_{k}(x, z)$ analytic in $(x, z)$ and $\left\|r_{k}(x, z)\right\| \leq d \int_{c}^{\infty} g(t) d t$ for a constant $d$ and $c \geq a, \forall(x, z) \in[c, \infty) \times \Omega$ and where the constant $d$ only depends on the constants from the dichotomy condition. In particular, by taking c large enough, one can get arbitrarily small bounds on $r_{k}$, that is, $r_{k}(x, z) \rightarrow 0$ as $x \rightarrow \infty$.

The proof follows closely that of Theorem 3.5 in [6]. This result thus shows the form of eigenvalue solutions obtained when (2.4) is reduced into Levinson's form. Thus in order to reduce (2.4) into Levinson's form, one needs a $(I+Q)$ -

transformation [2, 10], standard Kummer-Liouville transformation [2] and a two diagonalisations in that order since we had assumed that the coefficients are twice differentiable.

The first $(I+Q)$-transformation performed on the system (2.4) helps to transform the conditionally integrable terms into integrable terms. But even after diagonalisations, if the largest part of the off diagonal terms of $R(2.6)$ are not integrable, one may still apply $(I+Q)$-transformations to transform them into integrable terms. Assume the matrix $\mathcal{C}$ and the coefficients admits a decomposition (1.2) and take

$$
\mathcal{C}_{1}+\mathcal{C}_{2}=\left[\begin{array}{cc}
A & B \\
C & A^{*}
\end{array}\right] \quad \text { and } \quad \mathcal{C}_{3}+\mathcal{C}_{4}=\left[\begin{array}{cc}
0 & 0 \\
C_{3}+C_{4} & 0
\end{array}\right] \text {. }
$$

For this, one needs $w_{3}=w_{4}=0$. This requirement is not necessary and all the following computations could be carried out without this assumption. The conditions, however, become rather technical. Now transform the system (2.4) by

$$
u(x)=F(x) v(x),
$$

with $F(x)=I+Q$. Thus one has

$$
\begin{aligned}
v^{\prime} & =\left[\mathcal{C}_{s}+(I+Q)^{-1}\left\{-Q^{\prime}+\mathcal{C}_{s} Q-Q \mathcal{C}_{s}+\mathcal{C}_{4}+\mathcal{C}_{4} Q\right\}\right. \\
& \left.+(1+Q)^{-1} C_{3}(I+Q)\right] v .
\end{aligned}
$$

If in equation (2.8) $Q^{\prime}=C_{4}, Q(x, z) \rightarrow 0$ as $x \rightarrow \infty$ and $C_{s} Q-Q C_{s}$ and $C_{4} Q$ are Levinson terms, the conditionally integrable terms have been eliminated effectively. In some cases, one can even choose $Q$ such that $Q^{\prime}=\mathcal{C}_{s} Q-Q \mathcal{C}_{s}+\mathcal{C}_{4}$. This will be the case if $\mathcal{C}_{s}$ is already diagonal. Hence choosing the simpler approach $Q=$ $-\int_{x}^{\infty} \mathcal{C}_{4}(t, z) d t$ will require that all the $p_{4}, q_{4}$ and $p_{0,4}$ be conditionally integrable. 
Additional constraints may also arise from the requirement that $\mathcal{C}_{s} Q-Q \mathcal{C}_{s}$ and $\mathcal{C}_{4} Q$ to be Levinson terms. After Kummer-Liouville (K.L)-transformations and two diagonalisations if the off diagonal terms of $R$ as mentioned earlier are not integrable, then one applies $(I+Q)$-transformations as follows. In this case let $\mathcal{C}_{s}=$ $\Lambda, \mathcal{C}_{3}+\mathcal{C}_{4}=R$ with $R_{i i}=0, i=1, \cdots, 2 n$, making the substitution $v=(1+Q) v_{1}$ equation (2.8) turns out to be

$$
v_{1}^{\prime}=\left(\Lambda+(I+Q)^{-1}\left(\Lambda Q-Q \Lambda+R-Q^{\prime}+R Q\right)\right) v_{1} .
$$

Since the diagonal part of $R$ is trivial by assumption, the equation $\Lambda Q-Q \Lambda+R=Q^{\prime}$ reduces to $(2 n-1) .2 n$ scalar equations, for which the solutions can be estimated well in terms of the coefficients $R_{i j}$. These equations are

$$
Q_{i j}^{\prime}=\left(\lambda_{i}-\lambda_{j}\right) Q_{i j}+R_{i j}, \quad i \neq j, \quad i, j=1, \cdots, 2 n .
$$

Necessary for this $(I+Q)$-transformation is $Q=o(1)$. Behncke [3] has shown, however, that the contribution to the diagonals as a result of $(I+Q)$-transformation are integrable and therefore irrelevant for spectral results.

The Kummer-Liouville (K.L)-transformation as used in the second order operators involved the change of variables but simplifies the expressions of the coefficients. In [1], the K.L-transformation was adapted to asymptotic integration. This transformation of (2.2) is formally derived from the transformation

$$
y(x)=\mu(x) z(t), \quad t=f(x) \quad \text { with } \quad f^{\prime}(x)=\gamma>0 .
$$

On the first order system level (2.4), this amounts to:

$$
u(x)=F(x) v(t), \quad t=f(x) \quad \text { with } \quad f^{\prime}=\gamma>0
$$

where $F$ is a $2 n$ by $2 n$ matrix. With this, the system equation (2.4) becomes

$$
\frac{d v}{d t}=\gamma^{-1}\left[F^{-1} \mathcal{C} F-F^{-1} F^{\prime}\right] v(t) .
$$

Because of the definition of the quasiderivatives (2.2), $F$ will consist of the coefficients of the differential equation (2.1), $\mu$, powers of $\gamma$ and their derivatives. For more details on how to compute $F$ explicitly, see [2]. In our case a standard K.Ltransformation will suffice. This will amount to $\left(w_{1}+w_{2}\right)^{\frac{1}{2 n}}=1$ [2].

Since the first $(I+Q)$-transformation will be followed by K.L-transformation, one needs the following smoothness conditions

$$
\begin{array}{ccc}
\mu^{2} \gamma^{2 k} p_{3}, & \mu^{2} \gamma^{2 m-1} q_{3}, & \mu^{2} \gamma^{-1} p_{0,3}, \\
\mu^{2} \gamma^{2 k+1} \widehat{p}_{4}, & \mu^{2} \gamma^{2 m} \widehat{q}_{4}, & \mu^{2} \widehat{p}_{0,4} \in \mathcal{L}^{1} .
\end{array}
$$

These conditions will guarantee that the $(I+Q)$-transformation and subsequent K.L-transformations map the perturbation terms $p_{3}+p_{4}, q_{3}+q_{4}, p_{0,3}+p_{0,4}$ into 
Levinson terms with respect to the variable $t$. Thus the condition for $(I+Q)$ back-transformation are

$$
\gamma^{2 k+2} \widehat{p}_{k, 4} w^{-1}, \quad \gamma^{2 m+1} \widehat{q}_{4} w^{-1}, \gamma \widehat{p}_{0,4} w^{-1} \rightarrow 0 .
$$

In order to diagonalise (2.4), one needs the eigenvalues of (2.4). Expansion of $\operatorname{det}(\mathcal{C}-\lambda .1)=\mathcal{P}$ and substituting the eigenvalue parameters $\lambda$ by $-i \lambda$, although this should be kept in our mind throughout and will be used without mentioning, leads to

$$
\mathcal{P}_{F}(x, z, \nu)=\sum_{k=0}^{n} p_{k} \lambda^{2 k}+2 \sum_{j=1}^{n} q_{j} \lambda^{2 j-1}-z w
$$

in case of (2.1) and will be called Fourier characteristic polynomial. This is a polynomial with real coefficients if $z$ is real, reflecting the symmetry of $L$. The characteristic polynomial of (1.1) thus becomes

$$
\mathcal{P}=\lambda^{2 n}+p \lambda^{2 k+1}+q \lambda^{2 m}+p_{0}-z .
$$

Necessary and sufficient conditions for the eigenvalues of (2.15) to be in three groups of different magnitudes, that is, small, intermediate and large eigenvalues such that $\left|\lambda_{s m}\right| \ll\left|\lambda_{\text {int }}\right| \ll\left|\lambda_{l g}\right|$ is to demand that the coefficients $p, q$ and $p_{0}$ (with $z$ absorbed into $p_{0}$ ) satisfy

$$
|q|^{2(n-k)-1} \ll|p|^{2(n-m)} \quad \text { and } \quad|p|^{2 m} \cdot\left|p_{0}\right|^{2(k-m)+1} \ll|q|^{2 k+1}
$$

so that the measure of the magnitude of the roots are given by

$$
\begin{gathered}
\left|\lambda_{l g}\right| \approx m_{l g}=|p|^{\frac{1}{2 n-2 k-1}}, \quad\left|\lambda_{i n t}\right| \approx m_{i n t}=\left|\frac{q}{p}\right|^{\frac{1}{2 k-2 m+1}}, \\
\left|\lambda_{s m}\right| \approx m_{s m}=\left|\frac{p_{0}}{q}\right|^{\frac{1}{2 m}} .
\end{gathered}
$$

This (2.16) implies $m_{l g} \gg m_{\text {int }} \gg m_{s m}$ and thus the roots of (2.15) can be approximated by iteration. Thus write (2.15) into the following three iteration schemes

$$
\begin{gathered}
\lambda_{l g}^{2 n-2 k-1}=-p+\mathcal{R}_{l g}(\lambda), \quad \lambda_{\text {int }}^{2 k-2 m+1}=-\frac{q}{p}+\mathcal{R}_{\text {int }}(\lambda), \\
\lambda_{s m}^{2 m}=-\frac{p_{0}}{q}+\mathcal{R}_{s m}(\lambda),
\end{gathered}
$$

where $\mathcal{R}_{l g}(\lambda)=-q \lambda_{l g}^{2 m-2 k-1}-p_{0} \lambda_{l g}^{-2 k-1}, \mathcal{R}_{i n t}(\lambda)=-\frac{p_{0}}{p} \lambda_{i n t}^{-2 m}-\frac{\lambda_{i n t}^{2 n-2 m}}{p}$ and $\mathcal{R}_{s m}(\lambda)=-\frac{p}{q} \lambda_{s m}^{2 k+1}-\frac{\lambda_{s m}^{2 n}}{q}$. For $m_{s m}, m_{i n t}$ and $m_{l g}$ to be the measure of magnitude of the eigenvalues, we have to show that $\mathcal{R}_{l}(\lambda), l=s m$, int, $l g$ is small. Thus 
Lemma 2.2. Assume $p, q$ and $p_{0}$ satisfy (2.16) such that $m_{\text {sm }}, m_{\text {int }}$ and $m_{l g}$ are given by $(2.17)$ then $\mathcal{R}_{l}(\lambda)=O(\eta)$ where $\eta=\max \left(\frac{m_{s m}}{m_{\text {int }}}, \frac{m_{\text {int }}}{m_{l g}}\right)$ is a continuous function and $\eta=o(1)$ uniformly as $x \rightarrow \infty$.

Proof. It is enough to show this only for $\mathcal{R}_{l g}(\lambda)$ since the rest can be proved in a similar fashion. Now write

$$
\mathcal{R}_{l g}(\lambda)=-q|p|^{\frac{2 m-2 k-1}{2 n-2 k-1}}-p_{0}|p|^{\frac{-2 k-1}{2 n-2 k-1}} .
$$

It suffices to show that each summand of this equation is $O(\eta)$. Thus we have

$$
|q \| p|^{\frac{2 m-2 k-1}{2 n-2 k-1}}=|q|^{-\frac{2 m}{2 k-2 m+1}}|p|^{\frac{2 k+1}{2 k-2 m+1}}|p|^{\frac{2 m}{2 n-2 k-1}}\left(\frac{m_{i n t}}{m_{l g}}\right)^{2 k+1}=O(\eta)
$$

and the second summand gives

$$
\left|p_{0}\right||p|^{\frac{-2 k-1}{2 n-2 k-1}}=\left|p_{0}\right|^{\frac{2 m-2 k-1}{2 m}}|q|^{\frac{2 k+1}{2 m}}\left(\frac{m_{s m}}{m_{l g}}\right)^{2 k+1}=O(\eta) .
$$

Since by (2.16) $m_{l g} \gg m_{\text {int }} \gg m_{s m}$, it implies that $\eta=o(1)$ as $x \rightarrow \infty$.

Theorem 2.3. For any root $\lambda$ of the polynomials in (2.18) there is a root $\widetilde{\lambda}$ of $\mathcal{P}$ (2.15) with $|\lambda-\widetilde{\lambda}|=O(\eta)$

Proof. Let $\lambda_{0}$ be a fixed root of any of the polynomials in (2.18). Writing each of them in their full expression say, for large eigenvalues, $\mathcal{P}_{l g}=\lambda_{l g}^{2 n-2 k-1}+p-\mathcal{R}_{l g}(\lambda)$, and equating $\mathcal{P}(2.15)$ to zero amounts to

$$
\left(\lambda-\lambda_{0}\right)=-\frac{\mathcal{R}_{l}(\lambda)}{Q_{l}(\lambda)}
$$

where $Q_{l}(\lambda)$ is one of the factors of the polynomial $\mathcal{P}_{l g}$. Near $\lambda=\lambda_{0}$ the right hand side is analytic in $\lambda$ and can be estimated uniformly in $x$ by $\eta(x)$. Since also $\partial_{\lambda}\left(\frac{\mathcal{R}_{l}(\lambda)}{Q_{l}(\lambda)}\right)$ can be estimated by $\eta$, a simple fix point argument proves the theorem.

By considering the resultant or the discriminant of $\mathcal{P}$ and $\partial_{\lambda} \mathcal{P}$, one can show that there are only finitely many spectral values $z$ for which $\mathcal{P}(\lambda, z)$ has multiple roots. Let $\omega_{1}<\omega_{2}<\cdots<\omega_{k}$ denote all of the real spectral values $z$ leading to multiple roots. Following [2], the analysis will be restricted to small complex neighbourhoods of $z_{0} \in\left(\omega_{i}, \omega_{i+1}\right), i=0, \cdots, k$, where $\omega_{0}=-\infty$ and $\omega_{k+1}=\infty$. For a given $z_{0} \in\left(\omega_{i}, \omega_{i+1}\right)$, one can now choose $\epsilon>0$ and $a>0$ so that $\mathcal{P}(\lambda, x, z)=$ 0 has no multiple roots for any

$$
z \in \mathcal{K}_{\epsilon}\left(z_{0}\right)=\left\{z|\quad| z-z_{0} \mid \leq \epsilon, \quad \operatorname{Im} z \geq 0\right\}=\mathcal{K}
$$

and $x \geq a$. This is possible because the roots of $\mathcal{P}$ depend analytically on the coefficients. Throughout the proof, it may be necessary to adjust $a$ and $\epsilon$ repeatedly 
and this will be done without mentioning. Thus $\mathcal{P}$ in (2.15) has $2 n$ distinct roots.

It is well-known that the matrix $T_{0}$ formed with the eigenvectors $\varrho(\lambda, x)$ of $\mathcal{C}$, $\mathcal{C}(x) \varrho(x, \lambda)=\lambda(x) \varrho(x, \lambda)$, diagonalises $\mathcal{C}$. The eigenvectors $\varrho$ of $\mathcal{C}$ for the eigenvalue $\lambda$ are given by, for $1 \leq k \leq n$,

$$
\begin{gathered}
\varrho_{k}=\lambda^{k-1} \\
\varrho_{n+k}=-i q_{k} \lambda^{k-1}+\sum_{r=k}^{n}(-1)^{k+r} p_{r} \lambda^{2 r-k}+\sum_{r=k}^{n-1}(-1)^{k+r} 2 i q_{r+1} \lambda^{2 r-k+1} .
\end{gathered}
$$

It is, however, advantageous to base the transformation $T$ (diagonalising matrix) on the eigenvectors $w_{k}=w\left(x, \lambda_{k}\right)$ ([10], Sections 3.1 and 3.3), where

$$
w_{k}(x)=M_{k}^{-\frac{1}{2}} \varrho\left(\lambda_{k}, x\right)=M_{k}^{-\frac{1}{2}}\left(1, \lambda_{k}, \lambda_{k}^{2}, \cdots\right), \quad M_{k}=\partial_{\lambda} \mathcal{P}\left(x, z, \lambda_{k}\right) .
$$

In this case, the measure of the size of $M$-factors, $M_{k}$, are given by

$$
\begin{gathered}
M_{l g} \approx|p|^{\frac{2 n-1}{2(n-k)-1}}, \quad M_{i n t} \approx|q|^{\frac{2 k}{2(k-m)+1}} \cdot|p|^{\frac{1-2 m}{2(k-m)+1}}, \\
M_{s m}=|q|^{\frac{1}{2 m}} \cdot\left|p_{0}\right|^{\frac{2 m-1}{2 m}} .
\end{gathered}
$$

It follows from the properties of $m_{s m}, m_{i n t}$ and $m_{l g}$ that $M_{l g} \gg M_{i n t} \gg M_{s m}$. Since the roots are distinct, $M_{k}$ is invertible $\forall k=s m$, int, $l g$. Thus, the transformation $T=\left(w_{1}, w_{2}, \cdots, w_{2 n}\right)$ and

$$
u=T v
$$

leads to

$$
v^{\prime}=\left(\Lambda-T^{-1} T^{\prime}\right) v \quad \text { with } \quad \Lambda=\operatorname{diag}\left(\lambda_{i}(x, z)\right) .
$$

The matrix elements of $T^{-1} T^{\prime}$ are given by $\left(T^{-1} T^{\prime}\right)_{i i}=0$, a consequence of the normalisation of the eigenvectors with the factors $M_{k}^{-\frac{1}{2}}$ leads to the following matrix elements in the general case

$$
\begin{aligned}
\left(T^{-1} T^{\prime}\right)_{j k} & =\left(\lambda_{k}-\lambda_{j}\right)^{-1} M_{j}^{-\frac{1}{2}} M_{k}^{-\frac{1}{2}}\left(\sum_{l=0}^{n}(-1)^{l} p_{l}^{\prime} \lambda_{k}^{l} \lambda_{j}^{l}\right. \\
& \left.-i \sum_{l=1}^{n}(-1)^{l} q_{l}^{\prime}\left(\lambda_{k}^{l} \lambda_{j}^{l-1}+\lambda_{k}^{l-1} \lambda_{j}^{l}\right)\right) .
\end{aligned}
$$

$[4,10]$. However, in the case of $(1.1)$, one can estimate the matrix elements of $T^{-1} T^{\prime}$ within the large, intermediate and small eigenvalue blocks by

$$
\frac{p^{\prime}}{p}, \quad \frac{q^{\prime}}{q}, \quad \frac{p_{0}^{\prime}}{p_{0}-z}
$$


with the corresponding antisymmetry. Thus from (2.21), the other nonzero matrix elements of $T^{-1} T^{\prime}$ can easily be approximated by

$$
\begin{aligned}
\left(T^{-1} T^{\prime}\right)_{l g, i n t} \approx & |p|^{-\frac{1}{2}}\left\{p^{\prime}\left(\left|\lambda_{i n t}\right|^{2 k+\frac{1}{2}-n}\left|\lambda_{i n t}\right|^{k}+\left|\lambda_{3}\right|^{2 k-n-\frac{1}{2}}\left|\lambda_{i n t}\right|^{k+1}\right)\right. \\
& \left.q^{\prime}\left|\lambda_{l g}\right|^{2 m-n-\frac{1}{2}}\left|\lambda_{i n t}\right|^{2 m-k}, \quad p_{0}^{\prime}\left|\lambda_{l g}\right|^{-n-\frac{1}{2}}\left|\lambda_{i n t}\right|^{-k}\right\}, \\
\left(T^{-1} T^{\prime}\right)_{l g, s m} \approx & |q|^{-\frac{1}{2}}\left\{p ^ { \prime } \left(\left|\lambda_{l g}\right|^{2 k+\frac{1}{2}-n}\left|\lambda_{s m}\right|^{2 k+\frac{1}{2}-m}\right.\right. \\
+ & \left.\left|\lambda_{l g}\right|^{2 k-n-\frac{1}{2}}\left|\lambda_{s m}\right|^{2 k+\frac{3}{2}-m}\right) \\
& \left.q^{\prime}\left|\lambda_{l g}\right|^{2 m-n-\frac{1}{2}}\left|\lambda_{s m}\right|^{m+\frac{1}{2}}, \quad p_{0}^{\prime}\left|\lambda_{l g}\right|^{-n-\frac{1}{2}}\left|\lambda_{s m}\right|^{-m+\frac{1}{2}}\right\}, \\
\left(T^{-1} T^{\prime}\right)_{i n t, s m} \approx & |q|^{-\frac{1}{2}}|p|^{-\frac{1}{2}}\left\{p ^ { \prime } \left(\left|\lambda_{i n t}\right|^{k}\left|\lambda_{s m}\right|^{2 k+\frac{1}{2}-m}\right.\right. \\
+ & \left.\left|\lambda_{i n t}\right|^{k-1}\left|\lambda_{s m}\right|^{2 k+\frac{3}{2}-m}\right) \\
& \left.q^{\prime}\left|\lambda_{2}\right|^{2 m-k-1}\left|\lambda_{1}\right|^{m+\frac{1}{2}}, \quad p_{0}^{\prime}\left|\lambda_{i n t}\right|^{-k-1}\left|\lambda_{s m}\right|^{-m+\frac{1}{2}}\right\}
\end{aligned}
$$

with the obvious antisymmetry. Thus if the coefficients are sufficiently smooth so that $T^{-1} T^{\prime}$ is small, the system (2.22) is almost diagonal. The matrix $T^{-1} T^{\prime}$ can be split up into a Levinson part $R$ and a smooth part, that is, differentiable remainder $S$. The Levinson part involves the coefficients $p_{2}^{\prime}, q_{2}^{\prime}$ and $p_{0,2}^{\prime}$. These are absolutely integrable by assumption. The differentiable part $S$ satisfies $S_{i j}(x, z) \rightarrow 0$ for $x \rightarrow \infty$ and $S_{i j}(x, z) \in \mathcal{L}^{2}$ uniformly for $z \in \mathcal{K}_{\epsilon}\left(z_{0}, \epsilon\right)$. The system (2.22) thus becomes

$$
v^{\prime}=(\Lambda+S+R) v \quad \text { with } \quad \Lambda=\operatorname{diag}\left(\lambda_{k}(x, z)\right)
$$

which can be diagonalised again. Thus, using change of variables to enable second diagonalisation, we have

$$
v_{1}=(I+B) v
$$

where $B_{i j}=\left(\lambda_{i}-\lambda_{j}\right)^{-1}\left(T^{-1} T^{\prime}\right)_{i j}$ with $B=o(1)$. Therefore, necessary for second diagonaliation we demand the following condition for elements within the blocks

$$
\left\{\left|\frac{p^{\prime}}{p}\right|, \quad\left|\frac{q^{\prime}}{q}\right|, \quad\left|\frac{p_{0}^{\prime}}{p_{0}-z}\right|,\right\}\left|\frac{q}{p_{0}-z}\right|^{\frac{1}{2 m}}=o(1) .
$$

Denoting the outside block elements by $\left(T^{-1} T^{\prime}\right)_{l g, i n t}=\xi_{3},\left(T^{-1} T^{\prime}\right)_{l g, s m}=\xi_{2}$ and $\left(T^{-1} T^{\prime}\right)_{i n t, s m}=\xi_{1}$, we therefore need similar conditions to (2.26) which is

$$
\left\{\xi_{1}, \quad \xi_{2} \quad \xi_{3}\right\}\left|\frac{q}{p_{0}-z}\right|^{\frac{1}{2 m}}=o(1) .
$$

Equations (2.26) and (2.27) arise from the small eigenvalue blocks only. The remainder terms after second diagonalisation can be approximated using the formula

$$
R_{1}=-(I+B)^{-1}\left(B^{\prime}+\left(T^{-1} T^{\prime}\right) B\right)
$$


and the leading contribution to the diagonal is thus given by

$$
\left.\Lambda_{2, i i}=-\sum_{j}\left(T^{-1} T^{\prime}\right)_{i j}\left(\lambda_{j}-\lambda_{i}\right)^{-1} T^{-1} T^{\prime}\right)_{j i} .
$$

The contributions resulting from (2.28) are integrable outside the small eigenvalue block. Critical for the $T$-back transformation are the solutions from the large eigenvalue and the $(B)_{l g, s m}$ matrix elements. In this case, one needs that

$$
M_{k}^{\frac{1}{2}} M_{j}^{-\frac{1}{2}} B_{j k}, \quad M_{k}^{\frac{1}{2}} M_{j}^{-\frac{1}{2}}\left(r_{k}\right)_{j}=o(1) .
$$

The asymptotic solution of (1.1) is then obtained on transforming back from $v_{1}$ to $u$. If $p, q$ and $p_{0}$ are twice differentiable, then after two diagonalisations and some $(I+Q)$-transformations, equation (1.1) has solutions given by

$$
\begin{aligned}
u_{k}(x, z)= & T(I+B))\left(I+Q_{1}\right) \cdots\left(I+Q_{l}\right) . \\
& .\left(e_{k}+r_{k}(x, z)\right) \exp \int_{a}^{x} \widetilde{\lambda}_{k}(t, z) d t,
\end{aligned}
$$

with $r_{k}(x, z) \rightarrow 0$ as $x \rightarrow \infty$ and $T$ being the diagonalising matrix obtained from the eigenvectors of $\mathcal{C} .(I+B)$ is the matrix for second diagonalisation arising from $(2.25)$ while $\left(I+Q_{r}\right)$ are matrices arising from the $(I+Q)$-transformations. $\widetilde{\lambda}$ is equal to $\lambda$ plus other contributions to the diagonals as a result of diagonalisations, for example, the contribution in (2.28). In order to analyse the expression (2.30), formally one shall also require that derivatives of higher order decay faster or they lead to integrable terms so that any additional correction term to the diagonals of the system as a result of transformations (diagonalisations and $(I+Q)$-transformations) will not affect the asymptotics of the solutions.

Somewhat, more concretely, one needs for the coefficients $f=p, q, p_{0}$

$$
\frac{f^{(k)}}{f} \in \mathcal{L}^{\frac{2}{k}} x^{-k \epsilon} \quad k=1,2 \quad \epsilon>0,
$$

that is, each derivative introduces a factor $\mathcal{L}^{2} x^{-\epsilon}$. If the coefficients are differentiable more often, this condition can be weakened in an obvious fashion. The consequences of such a smoothness and decay condition are

$$
Q_{j+1}=o\left(Q_{j}\right)=o(B) \quad r_{k}=o(B)
$$

so that the solution (2.30) can be simplified further. Since one is mainly interested in $y_{k}$ and its square integrability, one has to consider the first component of $u_{k}$, $\left(u_{k}\right)_{1}=y_{k}$ so that with $T_{1 k}=M_{k}^{-\frac{1}{2}}$

$$
y_{k}(x, z)=\left(M_{k}^{-\frac{1}{2}}+\sum_{j} M_{j}^{-\frac{1}{2}} B_{j k}\right)\left(e_{k}+r_{k}(x, z)\right) \exp \left(\int_{a}^{x} \widetilde{\lambda}_{k}(t, z) d t\right) .
$$


The term $M_{k}^{-\frac{1}{2}}+\sum_{j} M_{j}^{-\frac{1}{2}} B_{j k}$ (form factor) in standard situation is given by $M_{k}^{-\frac{1}{2}}$ but with unbounded coefficients and eigenvalues of different magnitude, the second term may be dominant. It also follows that the correction terms to the eigenvalues are integrable under normal circumstances but in case of slower decay and higher order smoothness this may not be true. In this study, however, we will assume that this is not the case and $\sum_{j} M_{j}^{-\frac{1}{2}} B_{j k}$ is square integrable whenever $M_{k}^{-\frac{1}{2}}$ is integrable.

\section{Dichotomy Condition}

Let $z$ be a spectral parameter such that $z=z_{0}+i \eta$ where $\eta>0$, then write the eigenvalues together with their correction terms as

$$
\lambda_{k}=\lambda_{k 0}+i \eta\left(\partial_{\lambda} \mathcal{P}\left(x, \lambda_{k}, z\right)\right)^{-1}, \quad \lambda_{k 0}=\lambda_{k}\left(z_{0}\right),
$$

where $\left(\partial_{\lambda} \mathcal{P}\left(x, \lambda_{k}, z\right)\right)^{-1}=M_{k}^{-1}[2]$.

Once the approximate values for the roots of the Fourier polynomial $\mathcal{P}(\lambda)$ and the $M$-factors are known, one has enough ingredients to establish the uniform dichotomy condition for the eigenvalues of the differential operator (1.1). The uniform dichotomy condition in Levinson's Theorem guarantees an $x$-uniform control of the unperturbed equation $u^{\prime}=\Lambda u$ which in some sense is also uniform in $\operatorname{Im} z$. Since the roots of $\mathcal{P}(x, \lambda, z)$ are calculated from characteristic Fourier polynomial $(2.15)$, the uniform dichotomy condition needed is equivalent to $\operatorname{sign} \operatorname{Im}\left(\lambda_{j}(x, z)-\lambda_{i}(x, z)\right)$ being constant modulo $\mathcal{L}^{1}$ for all unequal pair of indices $i$ and $j$. In spectral theory, a $z$-uniform dichotomy condition will be needed but that will only be relevant to small eigenvalues when $w \approx p_{0}$. In this study, slightly stronger conditions will suffice. Before the proof of dichotomy condition, one ! needs the following results.

Theorem 3.1. Let

$$
u^{\prime}=(\Lambda(x)+R(x)) u, \quad a \leq x \leq \infty, \quad \Lambda=\operatorname{diag}\left(\lambda_{1}(x), \cdots, \lambda_{2 n}(x)\right)
$$

be an asymptotically constant system of differential equations satisfying for $i=$ $1, \cdots, 2 n$

$$
\lambda_{i}(x)=\lambda_{i 0}+\lambda_{i 1}(x), \quad \text { with } \lambda_{i 0} \text { constant }
$$

$\lambda_{i 1} \rightarrow 0$ as $x \rightarrow \infty, \lambda_{i 1}$ continuous, $\lambda_{i 0}$ distinct, $R(x) \in \mathcal{L}^{1}$. Moreover assume $\operatorname{Re} \lambda_{i 0}=-\alpha, \alpha>0, i=1, \cdots, k$ and $\left|\operatorname{Re}\left(\lambda_{i 0}-\lambda_{j 0}\right)\right| \geq \delta>0, i=1, \cdots, k$, $j=k+1, \cdots, 2 n$. Then (3.2) has $k$ independent solutions

$$
u_{l}(x)=O\left(\exp \left(-\alpha x+\int_{a}^{x} m(t) d t\right)\right), \quad l=1, \cdots, k
$$

where $m(t) \rightarrow 0$ as $t \rightarrow \infty$.

Proof. Since $\mathcal{L}^{1}$-diagonal elements of $R$ can easily be transformed into integrable 
terms using $(I+Q)$-transformations and finally added to the diagonals of $\Lambda$, we may assume $R_{i i}=0, i=1, \cdots, 2 n$. Now choose $a \in \mathbb{R}$ so large that $\sum_{i, j=1}^{n} \int_{a}^{\infty}\left|R_{i j}(x)\right|$ $d x<\epsilon$, for $\epsilon>0$. $\epsilon$ will be fixed later. Let $m(x)=\max _{i=1, \cdots, k}\left(\operatorname{Re} \lambda_{i, 1}(x)_{+}\right)$with $f_{+}$denoting the positive part of $f$. We now follow the proof of Levinson's Theorem in Sect. 1.4 of Eastham's book [10]. First change variables by

$$
u(x)=v(x) \exp \left(-\alpha x+\int_{a}^{x} m(t) d t\right) .
$$

Then $v(x)$ satisfies

$$
v^{\prime}(x)=((\Lambda(x)+(\alpha-m(x)) \cdot 1)+R(x)) v(x)=\left(\Lambda_{1}+R\right) v
$$

with $\Lambda_{1}=\operatorname{diag}\left(\mu_{1}(x), \cdots, \mu_{2 n}(x)\right)$ satisfying $\left|\operatorname{Re}\left(\mu_{l}(x)-\mu_{j}(x)\right)\right| \geq \frac{\delta}{2}$ for $1 \leq l \leq k$, $j \leq k+1, \cdots, 2 n$. For $l \in\{1, \cdots, k\}$, however, we have $\mu_{l}(x) \leq 0$ and $\mu_{l}(x) \rightarrow 0$ as $x \rightarrow \infty$. Thus, let $P_{1}$ be the projection onto $e_{1}, \cdots, e_{k}$, the coordinate vectors $e_{j}$ for which $R e \mu_{j} \leq 0, x \geq a$. Let $\Phi(x)=\operatorname{diag}\left(\exp \int_{a}^{x} \mu_{i}(t) d t\right)$ be the fundamental matrix of the unperturbed system of (3.2), and let $\Phi_{1}=P_{1} \Phi$ and $\Phi_{2}=\left(I-P_{1}\right) \Phi$. Then for $l \leq k$ any solution $v$ of the integral equation

$$
\begin{aligned}
v(x) & =\Phi(x) e_{l}+\Phi(x) \int_{a}^{x} P_{1} \Phi^{-1}(t) R(t) v(t) d t \\
& -\Phi(x) \int_{x}^{\infty} P_{2} \Phi^{-1}(t) R(t) v(t) d t
\end{aligned}
$$

satisfies the differential equation. The existence of this solution $v(x)$ in (3.6) can thus be shown by iteration method with $v^{(0)}=\Phi e_{l}$

$$
\begin{aligned}
v^{(r+1)}(x) & =\Phi(x) e_{l}+\Phi(x) \int_{a}^{x} P_{1} \Phi^{-1}(t) R(t) v^{(r)}(t) d t \\
& -\Phi(x) \int_{x}^{\infty} P_{2} \Phi^{-1}(t) R(t) v^{(r)}(t) d t .
\end{aligned}
$$

Like in [10], there exists a constant $K$ such that

$$
\begin{gathered}
\left\|\Phi(x) P_{1} \Phi^{-1}(t)\right\| \leq K, \quad a \leq t \leq x<\infty \quad \text { and } \\
\left\|\Phi(x) P_{2} \Phi^{-1}(t)\right\| \leq K, \quad a \leq x \leq t<\infty .
\end{gathered}
$$

Choose $\epsilon>0$ and $a$ sufficiently large so that $K \epsilon \leq \frac{1}{8}$. By induction on $k$, one can easily show that $\left\|v^{(k)}(x)\right\|_{\infty} \leq 2$. Similarly, one sees as in Eastham's book [10], that

$$
\begin{aligned}
\left\|v^{(r+1)}(x)-v^{(r)}(x)\right\|_{\infty} & \leq(2 K \epsilon)\left\|v^{(r)}(x)-v^{(r-1)}(x)\right\| \\
& \leq(2 K \epsilon)^{r}\left\|v^{(1)}(x)-v^{(0)}(x)\right\| \\
& \leq 4^{-r}\left\|v^{(1)}(x)-v^{(0)}(x)\right\| .
\end{aligned}
$$


Thus, the sequence $\left(v^{r}\right)_{r=0}^{\infty}$ converges uniformly on $[a, \infty)$ to a solution $v_{l}$ of (3.2). From $\left\|v_{l}(a)-e_{l}\right\|_{\infty}<\frac{1}{3}$, we also see that all such solutions are linearly independent.

This Theorem has the following intepretation. Assume the roots of the polynomial $\mathcal{P}(\lambda, x, z)(2.15)$, for $z_{0} \in \mathbb{R}$, are

$$
\alpha_{1} \pm i \beta_{1}, \cdots, \alpha_{r} \pm i \beta_{r}, \gamma_{2 r+1}, \cdots, \gamma_{2 n}
$$

with $\alpha_{j}, \beta_{j}, \gamma_{l} \in \mathbb{R}$. Then the nonreal eigenvalues lead to $r$ square integrable solutions, which decay exponentially, because of the Theorem. This holds regardless of the dichotomy conditions. For the real eigenvalues, we have by implicit function theorem

$$
\gamma_{l}(z)=\gamma_{l}\left(z_{0}\right)+\left(\partial_{\lambda} \mathcal{P}\left(x, \gamma_{l}, z\right)\right)^{-1}\left(z-z_{0}\right) \text { for small } z-z_{0} .
$$

Thus the dichotomy condition holds if

$$
\left(\partial_{\lambda} \mathcal{P}\left(\gamma_{l}\right)\right) \neq\left(\partial_{\lambda} \mathcal{P}\left(\gamma_{m}\right)\right) \quad l \neq m,
$$

because $\left(\partial_{\lambda} \mathcal{P}(\lambda)\right)$ is real. $\gamma_{l}=\gamma_{l}\left(z_{0}\right)$ will then contribute to the deficiency index if $\left(\partial_{\lambda} \mathcal{P}\left(\gamma_{l}\right)\right)>0$, because the corresponding exponent is $i \gamma_{l}\left(z_{0}+i \eta\right) \approx$ $i \gamma_{l}\left(z_{0}\right)-\eta\left(\partial_{\lambda} \mathcal{P}\left(\gamma_{l}\left(z_{0}\right)\right)\right)^{-1}$. The associated eigenvalue solutions, however, will lose their square integrability as $\eta \rightarrow 0_{+}$if the coefficients are bounded. Since the signs of $\left(\partial_{\lambda} \mathcal{P}\left(\gamma_{l}\right)\right)$ are evenly distributed, half of the $\gamma$ will lead to square integrable solutions for $\eta=\operatorname{Im} z>0$ and the complementary $\gamma$ 's will lead to square integrable solutions in the lower half plane. This shows that for bounded coefficients with even number of eigenvalues say $2 n, L$ is limit point and $\operatorname{def} L=(n, n)$. If the coefficients are unbounded, some of the solutions related to $\gamma$-eigenvalues may stay square integrable as $\operatorname{Im} z \searrow 0$. In this case the deficiency indices may be $\operatorname{def} L=(n+d, n+d)$, $0 \leq d \leq n$ if $d$ solutions of $L y=z y$ associated to real roots of $\mathcal{P}(x, \lambda, z))$ stay square integrable. But in all cases, it suffices to check the dichotomy condition only for the real roots of $\mathcal{P}(x, \lambda, z))$, see [7].

Theorem 3.2(Bezout's Theorem, [12] Theorem 7.5). Suppose two plane algebraic curves $C_{1}$ and $C_{2}$ have no common curve components (that is, the polynomials defining $C_{1}$ and $C_{2}$ have no common factor). Then, their intersection number is given by

$$
\left(C_{1} \cdot C_{2}\right)=\sum_{p \in C_{1} \cap C_{2}}\left(C_{1} \cdot C_{2}\right)_{p}=\operatorname{deg} C_{1} \cdot \operatorname{deg} C_{2} .
$$

Bezout's theorem implies that two irreducible algebraic curves intersecting in infinitely many points are identical. That is, two distinct irreducible algebraic curves intersect at most in finitely many points.

In the next lemma, Lemma 3.3, the eigenvalues will be considered as functions of $x$ and $z$. 
Lemma 3.3 Assume that

(i) the eigenvalues of each eigenvalue group, that is, large, intermediate and small, are distinct,

(ii) the nonreal eigenvalues of each eigenvalue group have an imaginary part $\operatorname{Im} \lambda_{j} \approx m_{j}$, where $j=l g$, int, sm and the real roots $\lambda$ and $\mu$ of the same eigenvalue group $j$ satisfy $\partial_{\lambda} \mathcal{P}(\lambda) \neq \partial_{\lambda} \mathcal{P}(\mu) \quad \lambda \neq \mu$,

(iii) the group of small eigenvalues $\lambda_{\text {sm }}$ satisfy (i) and (ii) if $w \ll p_{0}$ and satisfy $z$-uniformly dichotomy condition if $w \approx p_{0}$.

Then the eigenvalues of the Fourier polynomial (2.15) satisfy the uniform dichotomy condition.

Proof. The proof is divided into two parts, that is, the dichotomy condition between eigenvalues of different groups and the dichotomy condition between the eigenvalues of the same group.

Eigenvalues of different groups

Three cases arise when one considers (3.1). Assume $l$ and $m$ denote different groups of large, intermediate and small eigenvalues such that $\left|\lambda_{l}\right| \gg\left|\lambda_{m}\right|$. Then

a) $\operatorname{Im} \lambda_{l 0} \approx m_{l}, \operatorname{Im} \lambda_{m}$ arbitrary.

The dichotomy condition is satisfied because $m_{l} \gg m_{m}$,

b) $\operatorname{Im} \lambda_{l 0}=\operatorname{Im} \lambda_{m 0}=0$. The dichotomy condition is still satisfied, because $M_{l}^{-1} \ll M_{m}^{-1}$

c) $\operatorname{Im} \lambda_{l 0}=0, \operatorname{Im} \lambda_{m 0} \approx m_{m} \gg M_{m}^{-1} \gg M_{l}^{-1}$. The dichotomy condition is again satisfied.

Eigenvalues of the same group

Now assume that $\lambda(z)$ and $\mu(z)$ are eigenvalues of the same group and not from the small eigenvalue group. Then by Theorem 2.1, one only needs to check the dichotomy condition for real eigenvalues. But since $\lambda(z)$ and $\mu(z)$ satisfy the condition $\partial_{\lambda} \mathcal{P}(\lambda(z)) \neq \partial_{\lambda} \mathcal{P}(\mu(z))$ if $\lambda(z) \neq \mu(z)$ by assumption, it implies that $\lambda(z)$ and $\mu(z)$ have different slopes off the real axis. This is the required uniform dichotomy condition between $\lambda(z)$ and $\mu(z)$. If $w \ll p_{0}$, then the above reasoning applies to the small eigenvalue group too.

Now it remains to establish the $z$-uniform dichotomy condition within the group of small eigenvalues if $w \approx p_{0}$. In this case, the influence of large and intemediate eigenvalue groups to the small eigenvalue group will be assumed to be negligible and thus irrelevant. For the small eigenvalue group, the dichotomy condition is needed uniformly in $z$. Thus let $-\infty<\omega_{1}<\omega_{2}<\cdots<\omega_{k}<\infty$ be the enumeration of all the spectral values which lead to double roots of the Fourier polynomial (2.15) $\mathcal{P}$. Thus with $z \in \mathcal{K} \cap \mathbb{R}$ one can construct a set

$$
U_{1,2}=\left\{z \in\left(\omega_{1}, \omega_{i+1}\right) \mid \quad \frac{d \lambda_{1}(z)}{d z}=\frac{d \lambda_{2}(z)}{d z}\right\}
$$


for two distinct real roots of the small eigenvalue group. If $U_{1,2}$ is countable with accumulation points $\left(\omega_{i}, \omega_{i+1}\right)$, then one has uniform dichotomy condition satisfied for a real $z$ outside $U_{12}$, because $\lambda_{1}(z+i \eta)=\lambda_{1}(z)+i w \eta \frac{d \lambda_{1}(z)}{d z}$ with $\frac{d \lambda_{1}(z)}{d z}$ real by implicit function theorem. Since the eigenvalues from the small eigenvalue group depend analytically on $q$ and $p_{0}-z$, this extends immediately to $\lambda_{1}(z+i \eta)$ by continuity. This, however, is the required uniform dichotomy condition for $\lambda_{1}(z)$ and $\lambda_{2}(z)$ for the small eigenvalue group. If $U_{1,2}$ has accumulation point inside $\left(\omega_{i}, \omega_{i+1}\right)$, analyticity gives

$$
\frac{d \lambda_{1}(z)}{d z}=\frac{d \lambda_{2}(z)}{d z} \quad \text { or } \quad \lambda_{1}(z)=\lambda_{2}(z)+K
$$

for $z \in\left(\omega_{i}, \omega_{i+1}\right)$. Finally one has to show that the above relation is possible only if $K=0$. To see this, consider the algebraic curves $\mathcal{C}_{1}$ and $\mathcal{C}_{2}$ generated by the zeros of the polynomials $\mathcal{P}\left(\lambda_{1}, z\right)$ and $\mathcal{P}\left(\lambda_{2}, z\right)$ respectively. It is easy to see that $\mathcal{C}_{1}$ and $\mathcal{C}_{2}$ are irreducible because $\lambda_{1}$ and $\lambda_{2}$ appears linearly and the coefficient $q \neq 0$. The Riemann surfaces generated by $\mathcal{C}_{1}$, respectively $\mathcal{C}_{2}$, have a common segment since $\lambda_{1}(z)=\lambda_{2}(z)+K$ and hence by Bezout's Theorem $\mathcal{C}_{1}$ and $\mathcal{C}_{2}$ must agree and this can only be true if $\lambda_{1}(z)=\lambda_{2}(z)$ which again proves the uniform dichotomy condition for the small eigenvalue group.

For simplicity, we shall also require the coefficients to be power bounded and exclude the occurrence of logarithmic terms in the exponents, that is,

$$
p, q, p_{0}=O\left(x^{N}\right), \quad\left|\frac{w}{M_{k}}(x)\right| \leq C x^{-1-\epsilon} \quad \text { or } \quad\left|\frac{w}{M_{k}}(x)\right| \geq C x^{1+\epsilon},
$$

where $k$ is inteprated as $l g$, int and $s m$. This assumption avoids the interference of the form factors with the exponents. It can be avoided at the cost of further technical assumptions.

\section{Spectral Results}

Since the coefficients are unbounded, one is likely to have non-limit-point, that is, def $L=(n+r, n+r)$ thus we need a theorem on how the selfadjoint extension $H$ of $L$ can be defined and subsequently how to construct $M$-matrix, because it is the $M$-matrix that connects the asymptotics of the eigenvalue solutions with the spectrum of the selfadjoint realisation [15].

Theorem 4.1. Let $L$ be a formally symmetric differential operator of order $2 n$ on the interval $[a, \infty)$ for which $a$ is a regular boundary endpoint. Assume the deficiency index of $L$ is $(n+r, n+r)$. Then, the self adjoint extensions $H$ of $L$ with separated boundary conditions are defined by the domain

$$
\begin{gathered}
D(H)=\left\{y \in D\left(L^{*}\right) \mid\left(\alpha_{1}, \alpha_{2}\right) y(a)=0,\right. \\
\left.\lim _{x \rightarrow \infty} w_{k}^{*}(x) \mathcal{J} y(x)=0, \quad k=1, \cdots, r\right\} .
\end{gathered}
$$


Here $\alpha_{1}, \alpha_{2}$ satisfy the conditions in (2.5). The functions $w_{1}, \cdots, w_{r}$ are linearly independent modulo $D(L)$ at infinity and may be chosen as eigenfunctions of $L^{*} w=$ $z w, z \in \mathbb{C} \backslash \mathbb{R}$.

They also satisfy $\lim _{x \rightarrow \infty} w_{k}^{*}(x) \mathcal{J} w_{j}(x)=0$ for $j, k=1, \cdots, r$

Proof. The proof follows at once from ([18], Theorems 4.6, 5.4b and 5.5) and [15].

The functions $w_{k}$ are said to be linearly independent modulo $D(L)$ at infinity if there exists no non-trivial linear combination of corresponding modified functions $w_{j, \infty}$ which are identically zero in the neighbourhood of $a$ and for a sufficiently large $x$, the modified functions coincide with $w_{j}$ in $D(L)$.

Theorem 4.2. Let $L$ be a four term differential operator of the form (1.1). Assume that $p_{0}, p$ and $q$ admit decomposition (1.2), are real valued, locally integrable, twice differentiable and satisfy the condtions (2.12), (2.16), (2.26), (2.27), (2.30) and (3.9). Assume also $\sum_{j} M_{j}^{-\frac{1}{2}} B_{j k}$ is square integrable whenever $M_{k}^{-\frac{1}{2}}$ is integrable. Moreover, assume the coefficients $p_{0}$ and $q$ to be asymptotically constant when $w \approx p_{0}$.

(i) If $|p|^{\frac{2(m-n)+1}{2(n-k)-1}}$ is integrable, then $(\text { def } L)_{l g}=(n-k, n-k)$. The eigenvalue solutions that are square integrable are $z$-uniformly square integrable.

If $|p|^{\frac{2(m-n)+1}{2(n-k)-1}}$ is not integrable and $p>0$, then $(\operatorname{def} L)_{l g}=(n-k-1, n-k)$ and if $p<0$ then we have $(\operatorname{def} L)_{l g}=(n-k, n-k-1)$.

(ii) If $|q|^{\frac{-2 k}{2(k-m)+1}} \cdot|p|^{\frac{2(m-1)}{2(k-m)+1}}$ is integrable, then $k-m+1$ eigenvalue solutions are uniformly square integrable and $(\text { def } L)_{\text {int }}=(k-m+1, k-m+1)$.

If $|q|^{\frac{-2 k}{2(k-m)+1}} \cdot|p|^{\frac{2(m-1)}{2(k-m)+1}}$ is not integrable and if $\frac{q}{p}>0(q<0, p<0)$, then $(\operatorname{def} L)_{\text {int }}=((k-m),(k-m)+1)$ and if $\frac{q}{p}<0(q<0, p>0)$, then $(\operatorname{def} L)_{\text {int }}=(k-m+1, k-m)$.

Since the coefficients are unbounded, the small eigenvalue group will behave as those of a full operator since they depend analytically on $z$ and they are even in number, that is, $2 m$, thus

(iii) If $w \ll p_{0}$ then $(\operatorname{def} L)_{s m}=(m+1, m+1), \sigma(H)_{s m}$ is discrete if $|q|^{\frac{-1}{2 m}}$, $\left|p_{0}\right|^{\frac{-2 m+1}{2 m}}$ is integrable and $(\operatorname{def} L)_{s m}=(m, m), \sigma(H)_{s m}=\mathbb{R}$ if $|q|^{\frac{-1}{2 m}}$, $\left|p_{0}\right|^{\frac{-2 m+1}{2 m}}$ is not integrable.

If $p_{0} \approx w$, then the results of [2] apply, that is, there exists a constant $c$ such that $(\operatorname{def} L)_{s m}=(m, m),(-\infty, \underline{c}) \subset \sigma_{a c}(H, 1)_{s m}, \sigma_{\text {ess }}(H)_{s m} \cap[\bar{c}, \infty)=\emptyset$ and if $p_{0}(x) \rightarrow-\infty,(\text { def } L)_{s m}=(m, m)$ and $\sigma(H)_{s m}$ is discrete.

(iv) If $|p|^{\frac{2(m-n)+1}{2(n-k)-1}},|q|^{\frac{-2 k}{2(k-m)+1}} \cdot|p|^{\frac{2(m-1)}{2(k-m)+1}}$ and $|q|^{\frac{-1}{2 m}} \cdot\left|p_{0}\right|^{\frac{-2 m+1}{2 m}}$ are square integrable with $p_{0} \rightarrow \infty$ then $(\operatorname{def} L)=(n+2, n+2)$ and $\sigma(H)$ is discrete.

(v) If $|p|^{\frac{2(m-n)+1}{2(n-k)-1}},|q|^{\frac{-2 k}{2(k-m)+1}} \cdot|p|^{\frac{2(m-1)}{2(k-m)+1}}$ and $|q|^{\frac{-1}{2 m}} \cdot\left|p_{0}\right|^{\frac{-2 m+1}{2 m}}$ are not square integrable, $p \lessgtr 0, q \lessgtr 0, p_{0} \rightarrow \infty$ then $(\operatorname{def} L)=(n, n)$ and $\sigma_{a c}(H)=\mathbb{R}$ of multiplicity 2 . 
Proof. Let $L$ be $2 n$-th order differential operator generated by (1.1). Then by using quasiderivatives, the operator can be written in the first order system (2.4). An application of $(I+Q)$-transformation will eliminate conditionally integrable terms, that is $f_{4}$ where $f=p, q, p_{0}$. One then applies a standard K.L-transformation in order to obtain $w=1$. In order to bring the first order system into Levinson's form, one applies two diagonalisations since the smooth part of the coefficients are assumed to be twice differentiable. The system will be almost in diagonal form and if not, then the off diagonal elements that are conditionally integrable can be transformed into integrable terms by application of more $(I+Q)$-transformations. After all these transformations, the eigenvalue solutions will be given by (2.33).

The deficiency indices can thus be read off from the asymptotics of the eigenvalue solutions as $\operatorname{Im} z \searrow 0$. Thus assume $w M_{l g}^{-1}$ is integrable, then the solutions from the real eigenvalue is square integrable both in the upper and lower half planes and hence contributes to the deficiency indices. If $w M_{l q}^{-1}$ is not integrable, then this solution is integrable in the half plane if $p>0$ and fails to be integrable in the lower half plane. The situation is reversed when $p<0$. The deficiency indices will therefore be unequal. With a similar reasoning, the deficiency indices of the other groups of eigenvalues can be obtained.

Now assume that $w M_{l g}^{-1}, w M_{\text {int }}^{-1}$ and $w M_{s m}^{-1}$ are all integrable then all square integrable solutions are $z$-uniformly square integrable. Thus the solutions from real eigenvalues in all the three eigenvalue groups are $z$-uniformly square integrable, $L$ is non limit point and these solutions contribute at most to discrete spectrum only. The results of Theorem 4.1 can be applied for one to define a selfadjoint realisation $H$ of $L$. If $w M_{l g}^{-1}, w M_{\text {int }}^{-1}$ and $w M_{s m}^{-1}$ are not integrable, $p_{0} \rightarrow \infty$ while $p>0(p<0)$ and $q<0(q>0)$, then $\operatorname{def} L=(n, n)$ and thus selfadjoint extension of $L$ exists. Thus for spectral results, one needs the $M$-matrix. In order to construct the $M$-matrix, one then uses Remling's results [15].

Thus, let $Y_{\alpha}(., z)=\left(U_{\alpha}(., z), V_{\alpha}(., z)\right)$ be the fundamental matrix of (2.4) with initial values

$$
Y_{\alpha}(a, z)=\left[\begin{array}{cc}
\alpha_{1}^{*} & -\alpha_{2}^{*} \\
\alpha_{2}^{*} & \alpha_{1}^{*}
\end{array}\right], \quad \alpha_{1}, \alpha_{2} \quad \text { satisfy }(2.5) .
$$

$U_{\alpha}, V_{\alpha}$ are $2 n$ by $n$ complex-valued matrices whose every column solves $\tau u=z u$ and that $V_{\alpha}(., z)$ satisfy the self-adjoint boundary conditions at $a$. Therefore, the columns of $Y_{\alpha}(., z)$ span the $2 n$-dimensional vector space of solutions of (2.4).

Now fix the boundary conditions to the right through $\alpha=\left(\alpha_{1}, \alpha_{2}\right), \alpha_{1}=I$ and $\alpha_{2}=0$ and using the techniques of Hinton and Shaw [14], for $\operatorname{Im} z \neq 0$, the $M$-matrix, $M(z) \in \mathbb{C}^{n \times n}$ is defined by

$$
\chi(x, z)=Y_{\alpha}(x, z)\left[\begin{array}{c}
I_{n} \\
M(z)
\end{array}\right] \in \mathcal{L}^{2}[a, \infty) .
$$

$M(z)$ is analytic for $\operatorname{Im} z \neq 0$ and $\operatorname{Im} M(z)$ is positive definite in the upper half plane. The columns of $\chi(x, z)$ form a basis for the square integrable solutions of 
(2.4). Let $\chi(x, z)=\left[\begin{array}{l}W_{1}(x, z) \\ W_{2}(x, z)\end{array}\right]$ be $2 n$ by $n$ system of square integrable solutions of (2.4), then using Remling's results [15], there exists a regular $n$ by $n$ matrix $C$ such that

$$
Y_{\alpha}(x, z)\left[\begin{array}{c}
I_{n} \\
M(z)
\end{array}\right]=\left[\begin{array}{l}
W_{1}(x, z) \\
W_{2}(x, z)
\end{array}\right] C .
$$

Thus if one uses Dirichlet boundary conditions at $a$, one obtains

$$
M(z)=W_{2}(a, z) W_{1}^{-1}(a, z) .
$$

Now for the set $\mathcal{K}$, the solutions $\chi(., z)$ of the first order exist for all $z \in \mathcal{K}$ and uniformly continuous in $z$. This in turn shows that the spectrum is not singular continuous and apart from the isolated poles, the density of the spectral measures of $H$ is continuous. one thus has

$$
\lim _{\epsilon \rightarrow 0+} M(\mu+i \epsilon)=M(\mu+), \quad z=\mu+i \epsilon
$$

exists finitely and is bounded for all $z \in \mathcal{K}$. From the resolvent calculus of differential operators, it follows that the $M$-function is the Borel transform of the spectral measure. Thus the existence of a finite limit implies the absolute continuity of the corresponding spectral measure $\rho_{\alpha}$ and in fact $\frac{1}{\pi} \operatorname{Im} M(\mu+)$ gives the density of the absolutely continuous spectrum [15]. The rank of $\operatorname{Im} M(\mu+)$ is thus the multiplicity of $\sigma_{a c}(H)$. In this case $\operatorname{rank} \operatorname{Im} M(\mu+)=2$. It remains to extend the results to $x=0$ and other boundary conditions. For this, the results of Remling ([15], sect.6) can be applied. Thus $\mu$ belongs to the associated absolutely continuous spectrum if and only if there is at least one eigenvalue $\lambda(x, \mu)$, which for $x \rightarrow \infty$, its eigenvalue solution loses square integrability as $\operatorname{Im} z \searrow 0$. The multiplicity of the absolutely continuous spectrum is then given by the number of these eigenvalues.

Acknowledgement. The author would like to thank the anonymous referee for valuable comments that helped in improving the final version of this paper.

\section{References}

[1] H. Behncke, The Spectrum of Differential Operators with Almost Constant Coefficients II, J. Comput. Appl.Math., 148(2002), 287-305.

[2] H. Behncke, A Spectral Theory of Higher Order Differential Operators, Proc. London Math. Soc., 92(3)(2006), 139-160.

[3] H. Behncke, The Remainder in Asymptotic Integration II, to appear in Proc. Amer. Math. Soc.

[4] H. Behncke and D. B. Hinton, Eigenfunctions, Deficiency Indices and Spectra of Odd Order Differential Operators, Proc. London Math. Soc., (3) 97(2008), 425-449. 
[5] H. Behncke and C. Remling, Asymptotic Integration of Linear Differential Equations, J. Math. Anal. Appl., 210(1997), 585-597.

[6] H. Behncke and C. Remling, Uniform Asymptotic Integration of a Family of Linear Differential System, Math. Nachr., 225(2001), 5-17.

[7] H. Behncke, D. Hinton, and C. Remling, The Spectrum of Differential Operators of Order $2 n$ with Almost Constant Coefficients, Differential Equations, 175(2001), 130162.

[8] M. S. P. Eastham, The Deficiency Index of Even Order Differential Equations, J. London Math. Soc., 26(1982), 113-116.

[9] M. S. P. Eastham, Repeated Diagonalization and Extended Liouville-Green asymptotic Formulae, J. London Math. Soc., (2) 36(1987), pp 115.

[10] M. S. P. Eastham, The Asymptotic Solutions of Linear Differential Systems, London Math. Soc., Monographs New Series (Oxford University Press, Oxford), 1989.

[11] W. N. Everitt and D. Race, Some Remarks on Linear Ordinary quasi-differential Expressions, Proc. London Math. Soc., (3) 54(1987), 300-320.

[12] A. P. Griffiths, Introduction to Algebraic Curves, American Mathematic Soc., Beijing University Press., Beijing., 1989.

[13] D. B. Hinton and A. Schneider, On the Titchmarsh-Weyl Coefficients for Singular S-Hermitian Systems I, Math. Nachr., 163(1993), 323-342.

[14] D. B. Hinton and J. K. Shaw, On the Titchmarsh-Weyl M( $\lambda)$-Functions for Linear Hamiltonian Systems, J. Differential Equations, 40(1981), 316-342.

[15] C. Remling, Spectral Analysis of Higher Order Differential operator I, general properties of the M-Functions, J. London. Math. Soc., 58(2)(1998), 367-380.

[16] C. Remling, Spectral Analysis of Higher Order Differential operator II, Fourth Order Equations, J. London Math. Soc., 59(2)(1999), 188-206.

[17] P. W. Walker, A Vector-Matrix Formulation for Formally Symmetric Ordinary Differential Equations with Applications to Solutions of Integrable Square, J. London Math. Soc., 9(1974), 151-159.

[18] J. Weidmann, Spectral Theory of Ordinary Differential Operators, Springer Lect. Notes, 1258, Springer-Verlag, Berlin, 1987. 